\title{
Application of Cobalt Ferrite to an Ultra-Compact Magneto-Optical Isolator on Silicon Photonics Circuits
}

\author{
Y. SHOJI ${ }^{\text {a) }}$, M. YANAGA ${ }^{\text {a) }}$, T. KASHIMA ${ }^{\text {b) }}$, T. SUZUKI ${ }^{\text {b) }}$, S. NAKAGAWA ${ }^{\text {b) }}$, and T. MIZUMOTO ${ }^{\text {a) }}$ \\ a) Department of Electrical and Electronic Engineering, Tokyo Institute of Technology, 2-12-1-S9-9 Ookayama, Meguro-ku, \\ Tokyo 152-8552, Japan
}

b) Department of Physical Electronics, Tokyo Institute of Technology, 2-12-1-S3-42 Ookayama, Meguro-ku, Tokyo 152-8552, Japan

\begin{abstract}
A magneto-optical isolator is indispensable for optical fiber communication to protect active devices from unwanted reflections. A ferrite garnet such as yttrium iron garnet (YIG) is typically used because of its transparency and relatively large magneto-optic effect for near-infrared light $(\sim 0.8 \mathrm{eV})$. However, a YIG film must be epitaxially grown on a garnet substrate, which makes it difficult to integrate optical isolators with other photonic devices. In a literature, a cobalt ferrite has specific peak of magneto-optic effect at the infrared wavelength.

We then propose and design an ultra-compact magneto-optical isolator with cobalt ferrite on silicon photonic circuits. The isolator is based on a Mach-Zehnder interferometer of silicon optical waveguide fabricated on a silicon-on-insulator wafer. A cobalt ferrite film is partially deposited on the interferometer arms. In our design, the length of the nonreciprocal phase shifter is about $35 \mu \mathrm{m}$ which is on tenth of the case with Ce:YIG. We deposited cobalt ferrite films on buffer layer by a sputtering deposition. $\mathrm{A}(400)$-oriented $\mathrm{CoFe}_{2} \mathrm{O}_{4}$ film was fabricated on $\mathrm{FeO} / \mathrm{Fe}$ buffer layer to achieve low material loss.
\end{abstract}

Key Words: Spinel ferrite, Optical isolator

\section{INTRODUCTIONS}

In an optical fiber communication, backward reflected light induces unstable operation of laser diodes. An optical isolator is indispensable to eliminate such unwanted reflected light. Commercially available isolators are only based on bulk optics which is not suitable for integration. A waveguide optical isolator is required for integrated photonic circuits and devices.

Garnet ferrites having transparency and relatively large magneto-optic effect for near-infrared light with telecom wavelength of $1.55 \mu \mathrm{m}(0.8 \mathrm{eV})$ are typically used as the magneto-optic materials. A Ce-substituted yttrium iron garnet (Ce:YIG) film provides a Faraday rotation of $-4500 \mathrm{deg} / \mathrm{cm}$ and $\sim 3 \mathrm{~dB} / \mathrm{mm}$ absorption loss [1,2]. However, the single-crystalline Ce:YIG film must be epitaxially grown on a garnet substrate such as gadrinium garium garnet (GGG). Our group have investigated waveguide optical isolator with silicon photonic circuits realized by a direct bonding technique of silicon and Ce:YIG [3]. In that case, a Ce:YIG die grown on GGG substrate is directly bonded on a silicon photonic circuit without any adhesive. If a magneto-optic material can be deposited on silicon waveguide, integration of an optical isolator with other devices becomes much easier.

We then focus on a spinel ferrite $\mathrm{CoFe}_{2} \mathrm{O}_{4}$ which has specific peak of the Faraday rotation of $\sim 37000 \mathrm{deg} / \mathrm{cm} \mathrm{at} 0.8 \mathrm{eV}$ [4-6]. In this paper, we propose an application of a cobalt ferrite to a magneto-optical isolator. A poly-crystalline cobalt ferrite film can be deposited on silicon with thin buffer layers by a sputtering method. The film with single crystal plane may achieve lower optical loss than the literature.

\section{DEVICE STRUCTURE}

Figure 1 shows a schematic of the magneto-optical isolator with cobalt ferrite on silicon photonic circuits. The device is based on a Mach-Zehnder interferometer (MZI) of silicon waveguides. $3 \mathrm{~dB}$ couplers divide light into two waves with equal energies and couple the two waves. The MZI arms have nonreciprocal and reciprocal phase shifters. The nonreciprocal phase shifter has cobalt ferrite films on the waveguide where magnetic fields are applied in-plane and perpendicular to the light propagation direction (the Voigt configuration). Since the relations of light propagation and magnetization are opposite in two arms, it induces $-\pi / 2$ and $+\pi / 2$ phase differences for forward and backward directions, respectively. The reciprocal phase shifter having different optical path lengths induce $+\pi / 2$ phase difference for both the 
directions. Therefore, forward light propagating from the input port is induced constructive interference and transmitted to the output port, while backward light propagating from the output port is induced destructive interference and not coupled to the input port.

$\mathrm{Si}$ waveguides are fabricated on a silicon-on-insulator wafer. The $3 \mathrm{~dB}$ coupler and bending waveguide can be formed in several- $\mu \mathrm{m}$ footprint due to the strong light confinement of Si. The size of the isolator is dominated by the length of the nonreciprocal phase shifter. In our primary experiment, $\mathrm{CoFe}_{2} \mathrm{O}_{4}$ film without any buffer layer showed multiple peaks of $\mathrm{X}$-ray diffraction (XRD) measurement and the Faraday rotation was not detected from it. Multiple crystal planes in the poly-crystal $\mathrm{CoFe}_{2} \mathrm{O}_{4}$ might reduce the magneto-optic effect each other. Therefore, the actual waveguide structure should have buffer layer $\mathrm{FeO} / \mathrm{Fe}$ so that $\mathrm{CoFe}_{2} \mathrm{O}_{4}$ has single crystal plane. The amount of nonreciprocal phase shift is calculated by an optical mode solving and a perturbation theory [7]. Figure 2 shows the calculated nonreciprocal phase shift for the cases of $\mathrm{CoFe}_{2} \mathrm{O}_{4} / \mathrm{FeO} / \mathrm{Fe}, \mathrm{CoFe}_{2} \mathrm{O}_{4}$, and $\mathrm{Ce}: \mathrm{YIG}$ upper cladding layer on a Si waveguide with $0.5-\mu \mathrm{m}$ width. We used the Faraday rotation coefficients of $37000 \mathrm{deg} / \mathrm{cm}$ for the $\mathrm{CoFe}_{2} \mathrm{O}_{4}, 687000 \mathrm{deg} / \mathrm{cm}$ for $\mathrm{Fe}$ [8], and $-4500 \mathrm{deg} / \mathrm{cm}$ for the Ce:YIG layers, respectively. In order to find the effect of buffer layer, $\mathrm{CoFe}_{2} \mathrm{O}_{4}$ without buffer layer is also assumed to have the same Faraday rotation. The $\mathrm{FeO}$ is assumed to have no magneto-optic effect. The refractive indices of $\mathrm{CoFe}_{2} \mathrm{O}_{4}$, $\mathrm{FeO}$ and $\mathrm{Fe}$ are assumed to be $2.458+0.2034 \mathrm{i}, 2.32$, and 3.17+5.27i, respectively $[6,9,10]$. The thicknesses of $\mathrm{FeO}$ and $\mathrm{Fe}$ are both $10 \mathrm{~nm}$. The nonreciprocal phase shift with $\mathrm{CoFe}_{2} \mathrm{O}_{4} / \mathrm{FeO} / \mathrm{Fe}$ shows ten times larger than that with Ce:YIG. Due to the large Faraday rotation coefficient of $\mathrm{Fe}$, the nonreciprocal phase shift with $\mathrm{CoFe}_{2} \mathrm{O}_{4} / \mathrm{FeO} / \mathrm{Fe}$ increases slightly from that without $\mathrm{FeO} / \mathrm{Fe}$. However, the propagation loss becomes also higher due to the optical absorption of $\mathrm{Fe}$, therefore, the thickness of $\mathrm{Fe}$ should be thin as long as $\mathrm{CoFe}_{2} \mathrm{O}_{4}$ has single crystal plane. The minimum lengths of the nonreciprocal phase shifter are $35 \mu \mathrm{m}$ and $340 \mu \mathrm{m}$ for $\mathrm{CoFe}_{2} \mathrm{O}_{4} / \mathrm{FeO} / \mathrm{Fe}$ and $\mathrm{Ce}: \mathrm{YIG}$ cases, respectively.

However, the material loss of the $\mathrm{CoFe}_{2} \mathrm{O}_{4}$ measured in [6] is large. The propagation loss is estimated to be $6.8 \mathrm{~dB} / \mu \mathrm{m}$ when the nonreciprocal phase shift is maximized at the Si height of $0.17 \mu \mathrm{m}$. The loss is too high to obtain $\pm \pi / 2$ nonreciprocal phase difference. We then calculated the loss of the isolator for the shorter length of the nonreciprocal phase shifter which does not provide sufficient phase difference, but longer length of the reciprocal phase shifter can provide extinction peaks of the MZI output. That is, we ensure the extinction in the backward direction although the insufficient nonreciprocal phase shift results in excess loss of MZI in the forward direction. Figure 3 shows the total loss of the isolator as a function of the length of the nonreciprocal phase shifter. The MZI loss is dominant for the shorter length, on the other hand, the propagation loss is dominant for the longer length. The minimum total loss is $32.8 \mathrm{~dB}$ which is still high for the practical use. However, the assumed $\mathrm{CoFe}_{2} \mathrm{O}_{4}$ was fabricated by the conventional ceramic method [6]. So, we think that it should have included scattering and absorption losses due to some defects at the mismatch of crystal plane. Our fabrication approach is the use of sputtering method to achieve a film having a single crystal plane with good crystallinity. We may reduce the material loss less than the previous one. Also, Ref. [6] shows the reduction of the material loss by substituting $\mathrm{Fe}$ with $\mathrm{Al}$ to some amount. The refractive index and Faraday rotation of a $\mathrm{CoFe}_{1.4} \mathrm{Al}_{0.6} \mathrm{O}_{4}$ are

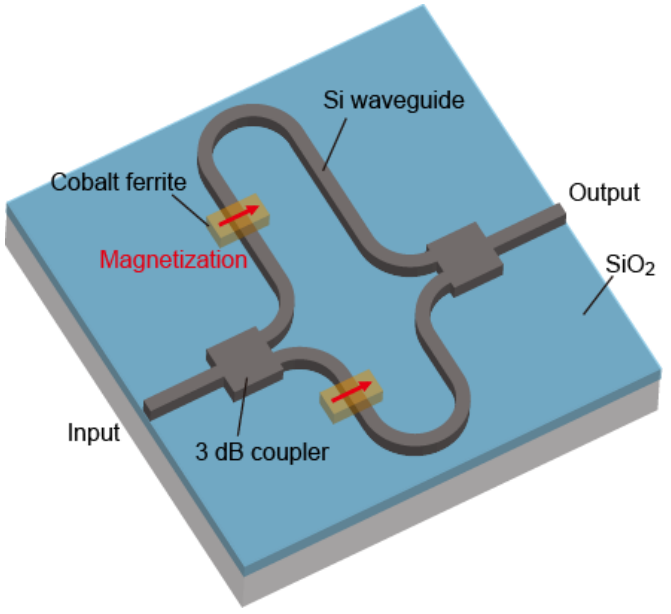

Fig. 1 Schematic of magneto-optical isolator with cobalt ferrite on silicon photonic circuits.

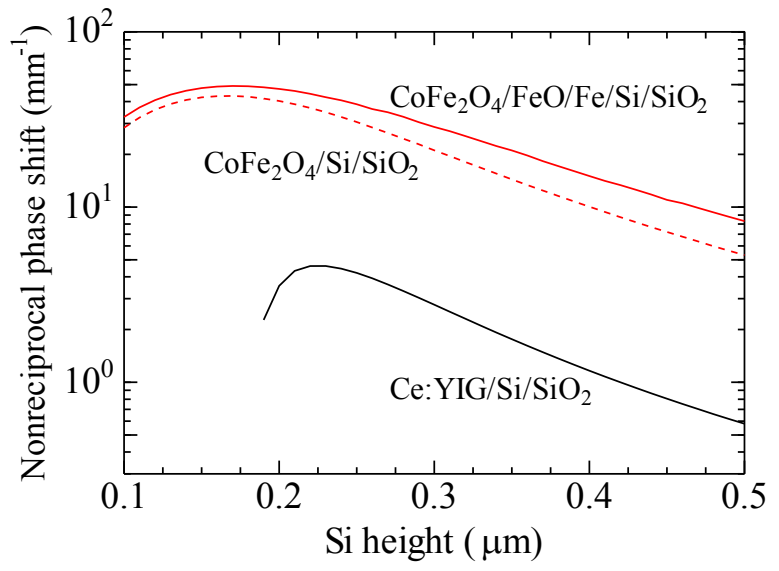

Fig. 2 Calculated nonreciprocal phase shift of $\mathrm{Si}$ waveguide with $\mathrm{CoFe}_{2} \mathrm{O}_{4} / \mathrm{FeO} / \mathrm{Fe}, \mathrm{CoFe}_{2} \mathrm{O}_{4}$ or $\mathrm{Ce}$ :YIG upper cladding layer. 


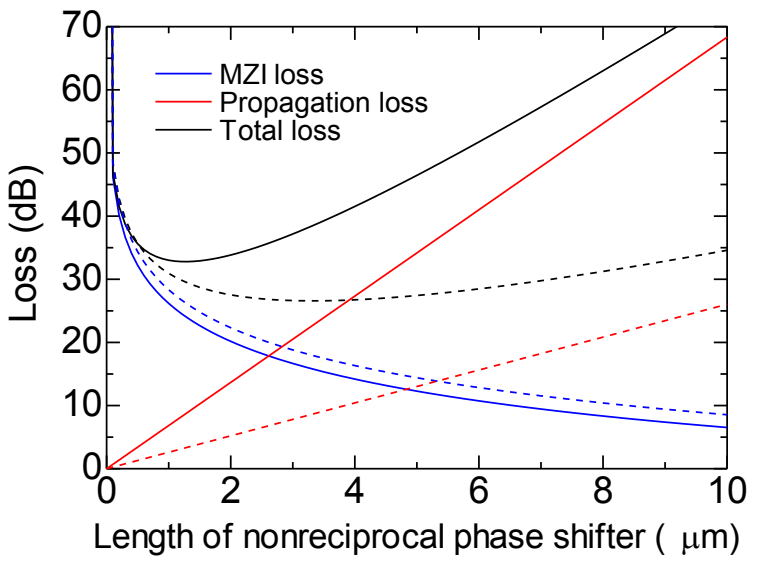

Fig. 3 Calculated loss of the isolator. Solid and dashed lines show the results for waveguide with $\mathrm{CoFe}_{2} \mathrm{O}_{4}$ and $\mathrm{CoFe}_{1.4} \mathrm{Al}_{0.6} \mathrm{O}_{4}$, respectively.

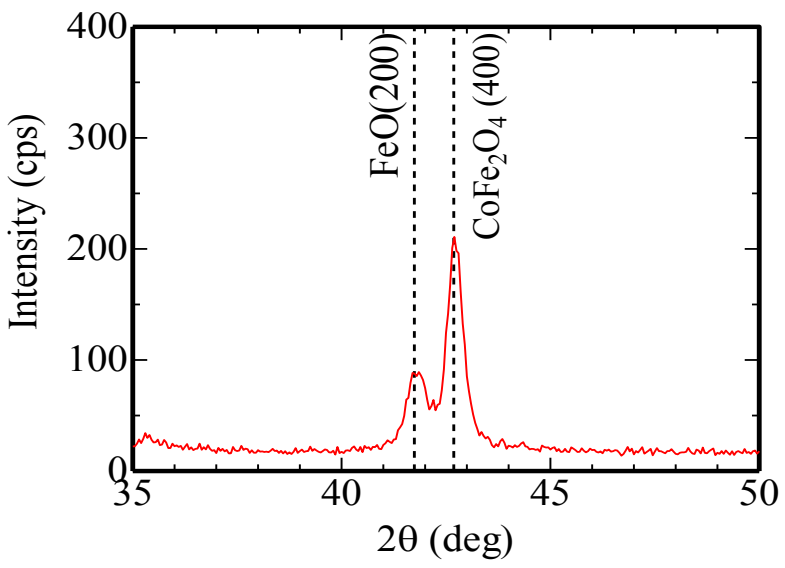

Fig. 4 X-ray diffraction spectrum of $\mathrm{CoFe}_{2} \mathrm{O}_{4} / \mathrm{FeO} / \mathrm{Fe}$ $\mathrm{FeO} / \mathrm{Fe}$ deposed on silica.

$2.169+0.076 \mathrm{i}$ and $32000 \mathrm{deg} / \mathrm{cm}$, respectively. The dashed line in Fig. 3 shows the calculated loss at the Si height of 0.13 $\mu \mathrm{m}$ using the properties of $\mathrm{CoFe}_{1.4} \mathrm{Al}_{0.6} \mathrm{O}_{4}$. The propagation loss is $2.6 \mathrm{~dB} / \mu \mathrm{m}$. The minimum loss is $26.6 \mathrm{~dB}$.

\section{DEPOSITION OF COBALT FERRITE}

Our cobalt ferrite film is fabricated by use of sputtering deposition with low temperature process. FeO/Fe buffer layer was deposited by an RF sputtering deposition on a silica glass. In this case, the thicknesses of $\mathrm{FeO}$ and $\mathrm{Fe}$ are $100 \mathrm{~nm}$ and $10 \mathrm{~nm}$, respectively. Then, $\mathrm{CoFe}_{2} \mathrm{O}_{4}$ film was deposited at $200{ }^{\circ} \mathrm{C}$ by an ion beam sputtering deposition.

Figure 4 shows the measured XRD spectrum of the cobalt ferrite film. Peaks of $\mathrm{CoFe}_{2} \mathrm{O}_{4}(400)$ plane and $\mathrm{FeO}(200)$ plane were clearly observed. However, Fe crystal plane was not observed because Fe was too thin to be crystallized. We think that a poly-crystalline cobalt ferrite film with single crystal plane is obtained.

\section{CONCLUSIONS}

We propose an application of cobalt ferrite to a magneto-optical isolator. By designing on silicon photonic circuits, an ultra-compact isolator can be realized. However, the material loss must be reduced less than the literature value. We deposited cobalt ferrite films on $\mathrm{FeO} / \mathrm{Fe}$ buffer layer by a sputtering method. The film with single crystal plane may reduce the material loss. In the next step, we have to reduce the thickness of $\mathrm{FeO}$ to be designed value, and investigate the deposition condition and film composition to acheive large Faraday rotation and low material loss.

\section{REFERENCES}

[1] M. Gomi, K. Satoh, and M. Abe, Jpn. J. Appl. Phys. 27, L1536 (1988).

[2] T. Shintaku and T. Uno, Jpn. J. Appl. Phys. 76, 8155 (1994).

[3] Y. Shoji, M. Ito, Y. Shirato, and T. Mizumoto, Optics Express 20, 18440 (2012).

[4] M. Abe and M. Gomi, J. Appl. Phys. 53, 8172 (1982).

[5] W. L. Peeters and J. W. D. Martens, J. Appl. Phys. 53, 8178 (1982).

[6] J. W. D. Martens, W. L. Peeters, P. Q. J. Nederpel, and M. Erman, J. Appl. Phys. 55, 1100 (1984).

[7] Y. Okamura, H. Inuzuka, T. Kikuchi, and S. Yamamoto, J. Lightwave Technol. LT-4, 711 (1986).

[8] G. S. Krinchik and V. A. Artemjev, J. Appl. Phys. 39, 1276 (1968).

[9] J. A. Dean, “Lange's Handbook of Chemistry 13th Edition”, McGraw-Hill Book Company, New York, U.S.A., 1985 , p.4.

[10] P. B. Johnson and R. W. Christy, Phys. Rev. B, 9, 5056 (1974). 